\title{
INVESTIGATION OF VACUUM ARC ANODE TEMPERATURES OF CU-CR AND PURE CU CONTACTS
}

\author{
St. Franke ${ }^{a}$,, R. Methling $^{a}$, S. Gortschakow ${ }^{a}$, M. Abplanalp ${ }^{b}$, \\ R.-P. Sütterlin ${ }^{b}$, T. Delachaux ${ }^{b}$, K. O. Menzel ${ }^{b}$ \\ a INP Greifswald, Felix-Hausdorff-Str. 2, 17489 Greifswald, Germany \\ ${ }^{b}$ ABB Corporate Research, ABB Switzerland, Segelhofstrasse 1K, 5405 Baden-Dättwil, Switzerland \\ * steffen.franke@inp-greifswald.de
}

\begin{abstract}
The present contribution reports on investigations of electrode temperatures for pure $\mathrm{Cu}$ electrodes and $\mathrm{Cu}-\mathrm{Cr}$ electrodes of different diameters exposed to vacuum arcs with sinusoidal currents of 5-15 kA and an axial magnetic field up to $180 \mathrm{mT}$. It is found that surface temperatures of pure $\mathrm{Cu}$ electrodes are significantly lower than for $\mathrm{Cu}-\mathrm{Cr}$ electrodes of the same diameter. This must be explained by different thermal properties of both materials. Reducing the diameter of $\mathrm{Cu}-\mathrm{Cr}$ electrodes it is found that surface temperatures increase, but moreover it is shown that the enthalpy stored in the electrode bulk material may effect electrode temperatures on timescales much longer than the current pulse width, particularly if there is no effective heat dissipation after current zero.
\end{abstract}

Keywords: vacuum arc, anode temperature, thermography.

\section{Introduction}

Surface temperatures of vacuum arc anodes are of dedicated interest in the research of vacuum arc interrupters, because they affect the switching capability of circuit breakers due to the temperature dependent evaporation of electrode material. Recently investigations on experimental determination of anode temperatures have been reported by means of a twotemperature pyrometer [1]. However, measurements where restricted to situations after current zero. In Dullni \& Schade 1993 [2] extensive investigations have been presented including calculation of the melt depth for different electrodes. A comparable approach was followed by Niwa et al. 2000 [3], which also considered the melt depth of electrodes. This contribution presents vacuum arc anode surface temperatures before and after current zero and discusses selected effects observed for different electrode materials and geometries.

\section{Experimental methods}

Three different types of electrodes have been investigated, namely pure $\mathrm{Cu}$ butt electrodes with $38 \mathrm{~mm}$ diameter, $\mathrm{Cu}-\mathrm{Cr} 50$ butt electrodes with $38 \mathrm{~mm}$ diameter, and $\mathrm{Cu}-\mathrm{Cr} 50$ pin electrodes with $10 \mathrm{~mm}$ diameter enclosed in Macor caps to restrict the arc attachment to the front surface. The upper electrode was fixed and the lower electrodes was drawn downwards by a pneumatic actuator up to an electrode distance of around $10 \mathrm{~mm}$. The distance from the electrode center to the observation windows was around $190 \mathrm{~mm}$, and the observation window diameter was about $150 \mathrm{~mm}$. The electrodes where exposed to sinusoidal currents of $5-15 \mathrm{kA}$. External axial magnetic fields up to $180 \mathrm{mT}$ have been applied to affect the constriction of

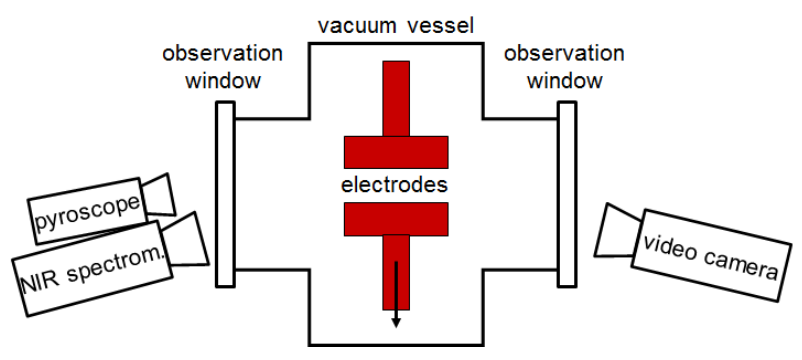

Figure 1. Experimental setup for investigation of vacuum arc anode temperatures (upper electrode). Three different optical detectors applied through observation windows: high-speed video camera, pyrometer and NIR spectrometer.

the arc. Hence, effects of different magnetic fields on anode temperatures could be investigated as well as effects of electrode material and electrode diameter.

Details of the experimental methods are described elsewhere [4. Nevertheless, the experimental procedure is summarized here. Three different detectors have been used to determine anode surface temperatures, namely high-speed video camera, pyrometer and near-infrared (NIR) spectrometer as represented in Fig. 1]

To reduce effects by metallization of observation windows, the observation window used for high-speed video imaging usually was displaced by a vacuum extender to a total distance from the electrode of about $500 \mathrm{~mm}$. The angle of inspection was therefore about $9^{\circ}$ from the horizontal. For pyrometer and IR spectrometer an angle of inspection of about $15^{\circ}$ was realized due to the shorter distance to the electrodes. In case of $\mathrm{Cu}-\mathrm{Cr}$ pin electrodes additional protective glasses have been introduced between the electrodes 
and the corresponding observation window, which could be exchanged without opening the vacuum vessel in case of significant window blackening by evaporated electrode material.

Near infrared spectroscopy was carried out by means of a fiber optics spectrometer in the wavelength range from 1000 to $1600 \mathrm{~nm}$ with a temporal resolution of $1-2 \mathrm{~ms}$ (Hamamatsu TG series C11482GA). The fiber optic spectrometer was focused by an imaging optics to the center of the anode surface. Due to the small angle of inspection the effective area was elliptic with an approximate dimension of $(0.4 \times 1.6) \mathrm{mm}$. The spectrometer has been calibrated by means of a tungsten strip lamp (OSRAM Wi $17 / \mathrm{G}$ ) in units of spectral radiance. Electrode temperatures after current zero were obtained by fitting Planck functions to spectral radiances of the radiating surfaces. For proper signals with high signal-to-noise ratio surface emissivities could be obtained as well.

The pyrometer (Kleiber 274 C LWL - High Speed Pyrometer) was focused to the anode surface by a long distance microscope (Questar LaVision QM 100). Pyrometric measurements were performed in the spectral range around $2 \mu \mathrm{m}$ with high temporal resolution $(<100 \mu \mathrm{s})$ but also without spatial resolution. Calibration was performed making use of the tungsten strip lamp at different currents and the calibrated NIR spectrometer. Application of the pyrometer to other materials than tungsten (like $\mathrm{Cu}-\mathrm{Cr}$ ) requires consideration of the emissivity of the material under investigation, and application to the active phase of the arc requires consideration of plasma background radiation. For these reasons absolute calibration was lost. However, relative calibration was maintained assuming temperature independence of emissivities. Finally, it was found that the pyrometer signal was dominated by thermal radiation after current maximum and that a characteristic feature in the temporal signal could be identified with a phase transition of the $\mathrm{Cu}-\mathrm{Cr}$ material. Pyrometer signals of $650 \mathrm{mV}$ therefore corresponded to the temperature of the eutectic point at $2020 \mathrm{~K}[5]$. Therefore absolute temperatures could be given with the pyrometer.

Two-dimensional spatio-temporal thermography could be realized by a high speed-video camera (Integrated design Tools Motion Pro Y4, 10000 frames per second) applying a Metal Interference Filter (MIF) at $890 \mathrm{~nm}$ with a full width at half maximum of $10 \mathrm{~nm}$. It was proven that there is no spectral line emission in this spectral range by a compact spectrometer (Avantes VIS-spectrometer, AvaSpec-ULS2048). Relative calibration was performed by calculating the Planck radiance transmitted by the MIF for different temperatures. Absolute calibration was matched to a pyrometer signal after current maximum (around $6 \mathrm{~ms}$ ). After removing spatial distortion 2D temperature maps could be obtained.

Before presentation of results and discussion a general remark has to be given. Occasionally it is referred

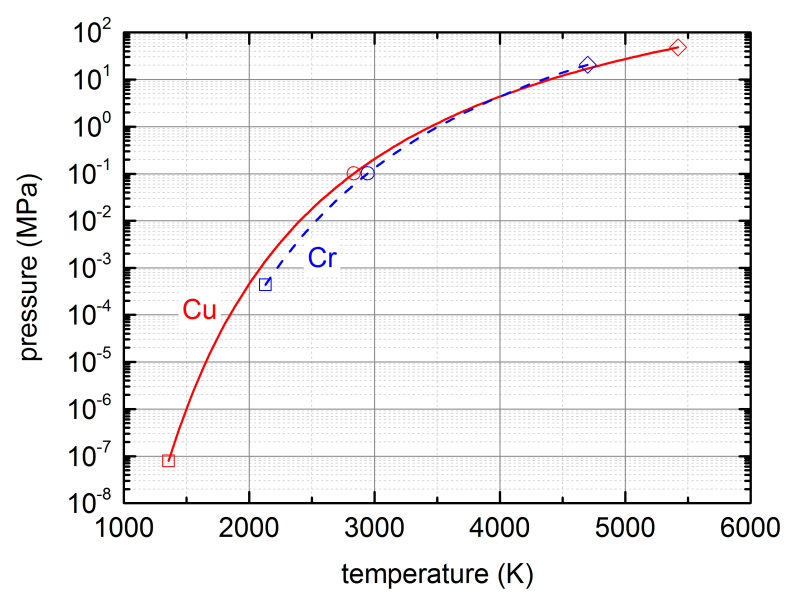

Figure 2. Vapor pressures of copper and chromium. Open squares mark the triple point, open circles the boiling point at standard pressure, and open diamonds the critical temperature.

to boiling temperatures of copper and chromium in the context of vacuum arc anodes. However, boiling temperatures depend on the ambient pressure. According to Clausius-Clapeyron the vapor pressure curve can be calculated, if the boiling temperature $T_{1}$ at $p_{1}=0.101325 \mathrm{MPa}$ standard pressure is given.

$$
\ln \left(\frac{p_{2}}{p_{1}}\right)=\frac{\Delta H_{m, v}}{R} \times\left(\frac{1}{T_{2}}-\frac{1}{T_{1}}\right)
$$

In this equation $R$ is the universal gas constant and $\Delta H_{m, v}$ is the molar enthalpy of evaporation, which is assumed to be a constant here. In Fig. 2 the vapor pressure curves for pure $\mathrm{Cu}$ and $\mathrm{Cr}$ are given [6] obtained from eqn. (1) making use of the boiling point at atmospheric pressure $(2835 \mathrm{~K}$ for pure $\mathrm{Cu}, 2944 \mathrm{~K}$ for pure $\mathrm{Cr}$ ). The vapor pressure curve is limited by the critical point at higher temperatures and the triple point at lower temperatures. The melting temperature is assumed to be independent on the pressure (1358 K for pure $\mathrm{Cu}, 2130 \mathrm{~K}$ for pure $\mathrm{Cr}$ ). Boiling occurs in an equilibrium situation, if the vapor pressure of the liquid is equal to the ambient pressure. In the context of vacuum arcs it is therefore not suggested to use the term boiling, as neither the equilibrium situation nor the ambient pressure are specifically given. For instance in front of the anode pressures around $0.003 \mathrm{MPa}$ are estimated in 7 , and simulations reveal pressures up to $0.04 \mathrm{MPa}$ [8]. However, within the scope of this contribution the vapor pressure curve in Fig. 2 demonstrates that up to atmospheric pressures the vapor pressure for $\mathrm{Cu}$ is significantly higher than for Cr.

\section{Results and discussion}

From NIR spectra temperatures are deduced by fitting Planck functions to the curves as exemplified in Fig. 3 for $\mathrm{Cu}-\mathrm{Cr}$ pin electrodes.

$$
L_{\lambda}(T)=\theta \varepsilon B_{\lambda}(T)
$$




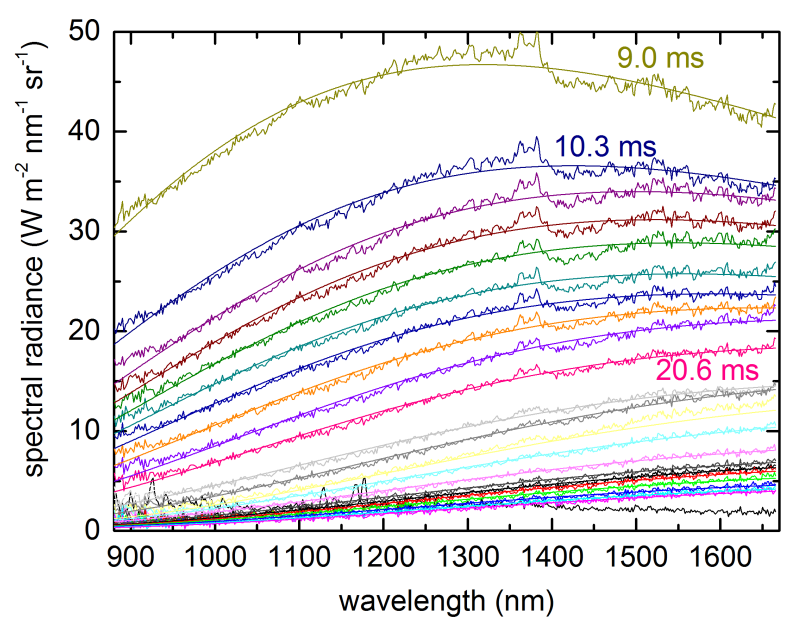

Figure 3. Planck fits to spectral radiances of $\mathrm{Cu}-\mathrm{Cr}$ pin electrodes after current zero.

Here $B_{\lambda}(T)$ denotes temperature and wavelength dependent Planck function, $\varepsilon$ emissivity of anode surface, and $\theta$ transmission of observation windows. For a vastly long time (up to $30 \mathrm{~ms}$ after current zero) Planck fits could be performed. Due to strong evaporation of electrode material during the active phase of the arc there is a significant reduction of observation windows transmission which is considered as time independent after current zero. The spectral dependence of transmission has been checked by independent measurements. Thermal radiance therefore is affected by both, surface emissivity and transmission, as given in the equation above. Due to the limited optical access no pyrometer data could be recorded.

Surface temperatures are given in Fig. 4. The statistical uncertainty is assumed to be $\pm 50 \mathrm{~K}$. Around current zero surface temperatures are close to the eutectic temperature of $\mathrm{Cu}-\mathrm{Cr}$ at $2020 \mathrm{~K}[5]$. For higher currents applied to the electrodes $10 \mathrm{~ms}$ after current zero there is a delay in the temporal temperature decay. The most reasonable explanation for this feature is to assume that the melt depth is significantly deeper for $10 \mathrm{kA}$ peak currents (see also [2]). Therefore the stored enthalpy in the electrode bulk material is higher and after an effective cooling period, immediately after current zero, excess heat is fed towards the electrode surface slowing down the temperature decay. This effect is potentially enhanced by the Macor cap which decreases heat dissipation of the electrodes towards the lateral surface of the cylindric pin electrode.

A similar effect has been obtained for $\mathrm{Cu}-\mathrm{Cr}$ butt electrodes as well. In Fig. 5 surface temperatures in the active phase and after current zero are given. The black dotted line represents an example of the sine-like current reaching current zero after $10 \mathrm{~ms}$. Solid lines show surface temperatures obtained from pyrometer and lines with symbols denote temperatures obtained from NIR spectrometer after current zero. For $\mathrm{Cu}-\mathrm{Cr} / 120 \mathrm{mT}$ one can see a plateau-like phase directly after current zero at temperatures between

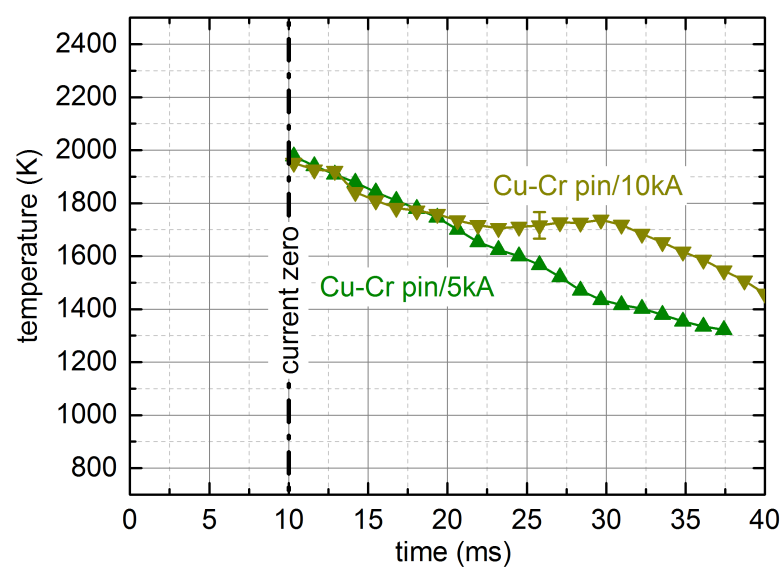

Figure 4. Anode surface temperatures for $\mathrm{Cu}-\mathrm{Cr}$ pin electrodes after current zero subjected to sinusoidal currents with $5 \mathrm{kA}$ and $10 \mathrm{kA}$ peak currents, respectively.

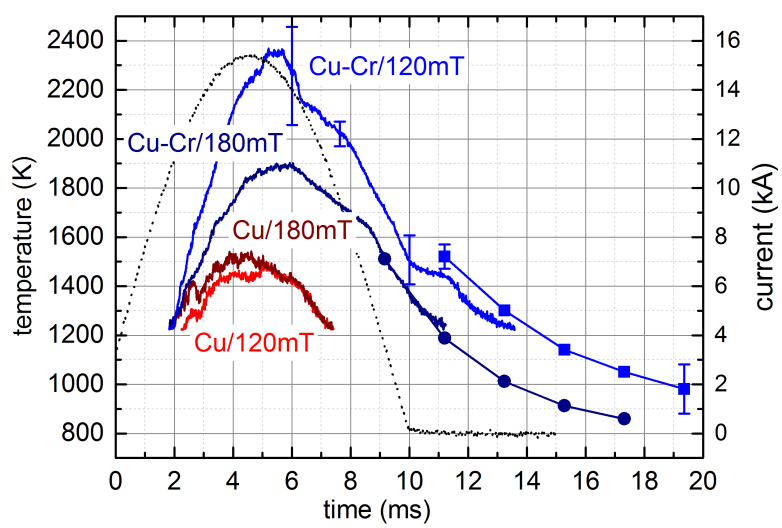

Figure 5. Anode surface temperatures for $\mathrm{Cu}-\mathrm{Cr}$ and pure Cu butt electrodes. Peak current is $15 \mathrm{kA}$ in all cases. The axial magnetic field varies between 120 and $180 \mathrm{mT}$. Black dotted line: current. Solid lines: temperatures obtained from pyrometer. Solid lines with symbols: temperatures obtained from NIR spectrometer.

1400 and $1600 \mathrm{~K}$. This kind of plateau has been found for several conditions, preferably at higher currents and lower magnetic fields, always after current zero but at different temperatures. The most reasonable explanation is again that due to the constricted arc attachment and local heating the melt depth was increased in these cases and excess heat in the bulk material slowed down the temperature decrease of the anode surface.

As expected higher magnetic fields avoid constriction of the arc attachment and hence lead to considerably lower surface temperatures. The agreement with temperatures from NIR spectroscopy are within experimental uncertainties, which are given in the figure. Lowest uncertainty is given around the eutectic point with a temperature of $2020 \mathrm{~K}$ marking a phase transition which is identified repeatedly with a pyrometer signal of $650 \mathrm{mV}$ and by a clear structure in almost 
all temporal courses of the surface temperature.

Surface temperature for pure $\mathrm{Cu}$ butt electrodes are much lower. There is almost no difference between the surface temperatures for the two different magnetic fields. This corresponds to high-speed videos which show a diffuse arc attachment for both reported cases. Reasons for the lower $\mathrm{Cu}$ temperatures are the higher thermal conductivity of $\mathrm{Cu}$ compared to $\mathrm{Cu}-\mathrm{Cr}$, lower melting temperature and hence stronger evaporation in liquid phase, which leads to additional cooling. No NIR spectra could be recorded for pure $\mathrm{Cu}$ electrodes after current zero due to low thermal emission.

One should keep in mind that only temperatures in the center of the electrodes are given up to now, due to the diagnostics discussed above. High-speed thermography is much more sophisticated, but $2 \mathrm{D}$ surface temperature distributions could be estimated from high-speed videos as given in Fig.6. This figure demonstrates that surface temperatures of $\mathrm{Cu}$ electrodes are not only significantly lower than for $\mathrm{Cu}-\mathrm{Cr}$ but furthermore $\mathrm{Cu}$ electrodes are much more homogeneously heated. In contrast the lower part of Fig. 6 shows an inhomogeneous distribution of surface temperatures for a $\mathrm{Cu}-\mathrm{Cr}$ electrode. This evidently demonstrates the necessity of $2 \mathrm{D}$ surface temperatures in case of constricted arc attachments to observe inhomogeneous temperature distributions.

\section{Summary}

Several methods for vacuum arc anode surface temperatures have been applied to different electrode materials and geometries. Vapor pressure curves propose massive evaporation of anode material, if the melting point is exceeded significantly. Furthermore it is argued that enthalpy stored in the bulk material of electrodes may affect surface temperatures, if they are heated at elevated levels in the active arc phase. This is partly in contradiction to previous publications which identified plateau-like phases of the temperature evolution by a phase transition [1, 3]. However, the present findings do not suggest a similar conclusion. Finally it is shown that 2D temperature distributions seem to be advantageous in case of constricted arc attachments, because they allow to conclude on the homogeneity of temperature distributions.

\section{References}

[1] Z. X. Wang, H. Ma, G. W. Kong, Z. Y. Liu, Y. S. Geng, and J. H. Wang. Decay Modes of Anode Surface Temperature After Current Zero in Vacuum Arcs - Part I: Experimental Study. IEEE Trans. Plasma Sci., 42(5):1464-1473, 2014.

doi:10.1109/TPS.2014.2316131

[2] E. Dullni and E. Schade. Investigation of High-Current Interruption of Vacuum Circuit-Breakers. IEEE Trans. Elec. Insu., 28(4):607-620, 1993. doi:10.1109/14.231543

[3] Y. Niwa, J. Sato, K. Yokokura, T. Kusano, E. Kaneko, I. Ohshima, and S. Yanabu. The Effect of Contact
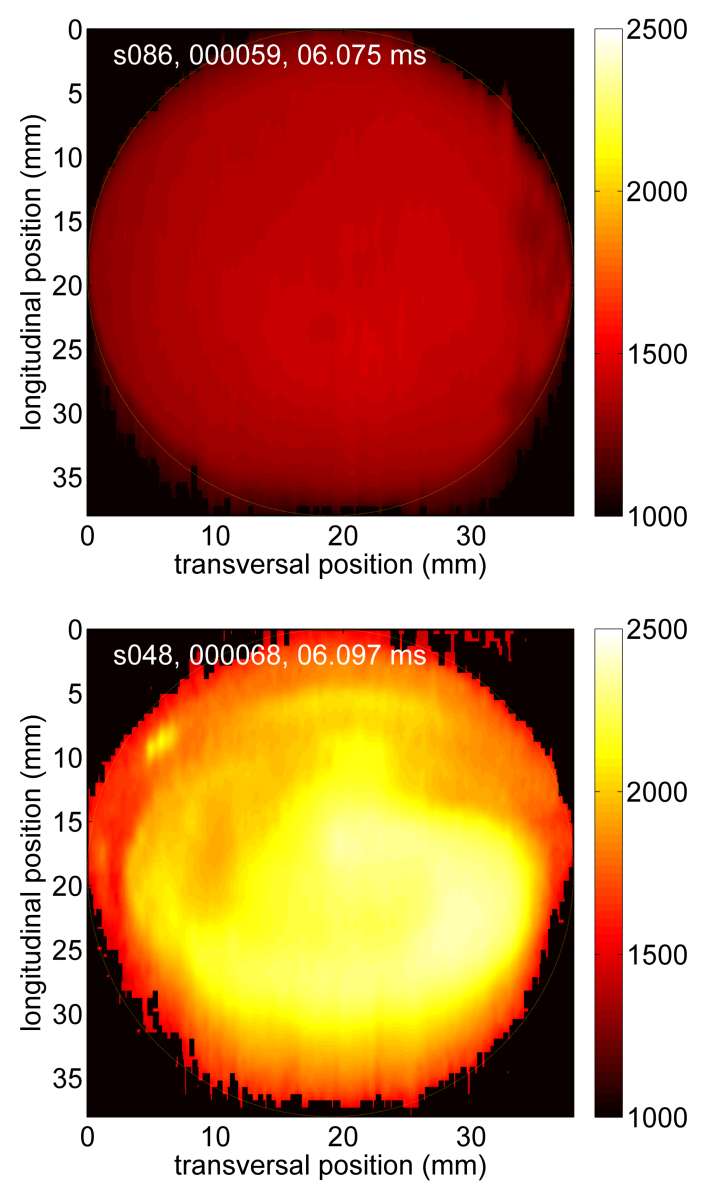

Figure 6. 2D anode surface temperature distribution around current zero at $15 \mathrm{kA}, 120 \mathrm{mT}$. Upper subfigure: pure Cu butt electrode. Lower subfigure: $\mathrm{Cu}-\mathrm{Cr}$ butt electrode.

Material on Temperature and Melting of Anode Surface in the Vacuum Interrupter. In Proc. 19th Int. Symp. on Disch. and El. Insul. in Vac., pages 524-527, Xi'an, China, 2000. doi:10.1109/DEIV.2000.879042.

[4] R. Methling, St. Franke, S. Gortschakow, M. Abplanalp, R.-P. Sütterlin, T. Delachaux, and K.O. Menzel. Comparison of methods of electrode temperature determination in high-current vacuum arcs. submitted to IEEE Trans. Plasma Sci., 2016.

[5] R. Müller. Arc-melted $\mathrm{Cu}-\mathrm{Cr}$ alloys as contact materials for vacuum interrupters. Siemens Forsch. Entw., 17(3):105, 1988.

[6] Dan Linder and Dean Linder. Chromium. URL: http://www.knowledgedoor.com/2/elements_ handbook/chromium.html

[7] R. Methling, S. Gorchakov, M. V. Lisnyak, S. Franke, A. Khakpour, S. A. Popov, A. V. Batrakov, D. Uhrlandt, and K. D. Weltmann. Spectroscopic Investigation of a Cu-Cr Vacuum Arc. IEEE Trans. Plas. Sci., 43(8):23032309, 2015. doi:10.1109/TPS.2015.2443856

[8] Y. Tian, Z. Wang, Y. Jiang, H. Ma, Z. Liu, Y. Geng, J. Wang, K. Nordlund, and F. Djurabekova. Modelling of crater formation on anode surface by high-current vacuum arcs. J. Appl. Phys., 120(18):183302, 2016. doi:10.1063/1.4967528 